\title{
Simultaneous Occult Breast Cancer and Rectum Cancer: A Case Report and Review of the Literature
}

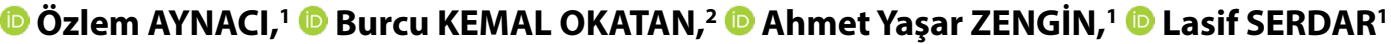 \\ 'Department of Radiation Oncology, Kanuni Training and Research Hospital, Trabzon-Turkey \\ ${ }^{2}$ Department of Pathology, Kanuni Training and Research Hospital, Trabzon-Turkey
}

\begin{abstract}
SUMMARY
Occult breast cancer $(\mathrm{OBC})$ and rectum cancer in the same patient is a rare event. Multiple primary tumors are being frequently reported in recent years owing to better screening programs, diagnostic procedures, and prolonged life span. In the case of a new cancer diagnosis, staging examinations are performed to evaluate the extent of disease, and sometimes a primary tumor is localized in cases where the metastatic area is a sign of malignancy. Adequate investigations, such as immunohistochemistry, must be performed in order to rule out metastasis of each other. We report the case of one patient diagnosed with $\mathrm{OBC}$ and rectum cancer synchronously in this case report.
\end{abstract}

Keywords: Immunohistochemistry; multiple primary tumors; occult breast cancer.

Copyright @ 2019, Turkish Society for Radiation Oncology

\section{Introduction}

Breast cancer on rare occasions can be detected only with isolated axillary mass without the presence of tumor on radiological and clinical examinations.[1] This rare presence is defined as occult breast cancer (OBC) by the American Joint Committee on Cancer, and it has been classified as T0, N0-1, M0, and stage II-III.[2] OBC is the second most common type of cancer after malignant lymphoma when the frequency of tumors that diagnosed with large axillary mass is examined.[3] The rate of OBC with axillary metastasis is $0.3 \%-1 \%$ of all breast cancers. Although the advances in imaging techniques have improved these rates over time, many cases still remain in the absence of primary location. Additionally, there is no consensus regarding its prognostic factor.[4]

Multiple primary tumors (MPTs) are defined as the occurrence of two or more unrelated tumors that originated from different organ sites at the same or different times.[5] MPTs have been first defined by Billroth in
1875 and modified by Warren and Gates in 1932.[6] Recently, there has been an increase in the number of patients diagnosed with MPTs. $[7,8]$ These malignancies that are observed at the same time or within are defined as synchronous MPTs, and these tumors that develop at more than a 6-month interval are defined as metachronous MPTs. [9]

The aim of the present study was to report an extremely rare case of simultaneous $\mathrm{OBC}$ and rectum cancer and to demonstrate our diagnostic approach and treatment procedure. We also emphasized the importance of immunohistochemical examination (IHC). This retrospective study includes only the patient's medical record and related images. Ethical approval was not thought to be necessary because the entire clinical course of the case was completely within the usual medical care. Written informed consent was obtained from the patient on each occasion of diagnostic examinations and therapeutic procedures and also for the publication of this case report. 


\section{Case Report}

A 76-year old woman was referred to our institution with a progressive complaint of left axilla lump. Her physical examination revealed that it was fluffy, necrotic, and $4-5 \mathrm{~cm}$ in size. She had no significant medical or family history. Her Karnofsky Performance Status was 70. No masses were palpable in neither the left nor the right breast. Histopathology of tru-cut biopsy showed that it is compatible with carcinoma invasion and metastasis, and it was recommended to investigate the systems particularly including the breast and gastrointestinal system (Fig. 1). IHC staining of tumor was positive $(+)$ for estrogen receptor $(E R)$ and progesterone receptor (PR) and negative (-) for human epidermal receptor-2 (HER-2).

She underwent positron emission tomography/ computed tomography (CT). A mass was displayed in her left axilla approximately $55 \times 70 \mathrm{~mm}$ in size with a high uptake of ${ }^{18}$ fluoro-2-deoxy-d-glucose 8 (FDG) with a standardized uptake value (SUV) of 16.69 and a high uptake of FDG with an SUV of 20.86 in the distal region of the rectum (Figs. 2 and 3).

The contrast-enhanced magnetic resonance imaging (MRI) of the breast showed that in the left axillary region, a limited diffusion mass with an intact appearance of $7 \times 11 \mathrm{~cm}$ irregularly shaped cystic necrotic area was observed. Specifically, no mass or non-mass enhancement was seen in the breast parenchyma (Fig. 4). Pathology specimens of axillary mass were re-examined after finding no apparent lesions in the breast MRI. IHC staining showed positivity for $\mathrm{ER}(+), \mathrm{PR}(+)$, gross cystic disease fluid protein 15 (GCDFP-15), and

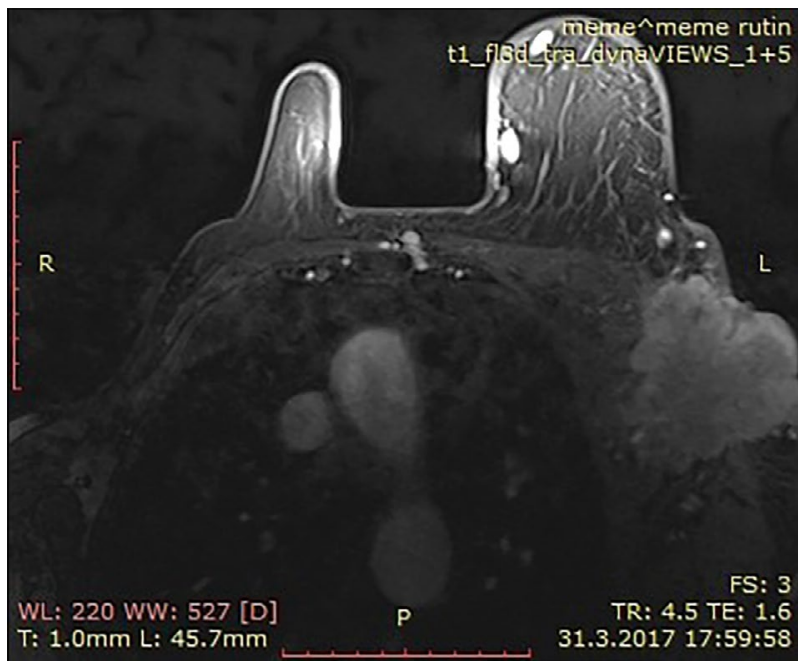

Fig. 1. Tumor infiltration of axillary biopsy, $10 \times$ magnification, hematoxylin \& eosin stain.

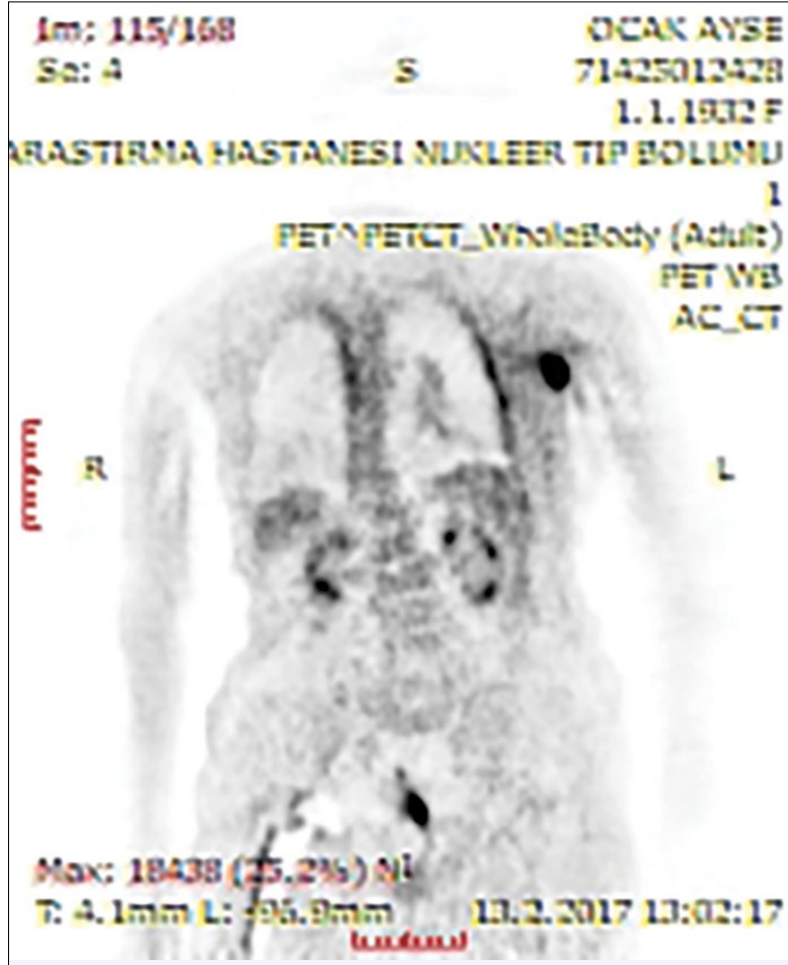

Fig. 2. PET coronal scan of the body shows a solid mass in her left axilla.

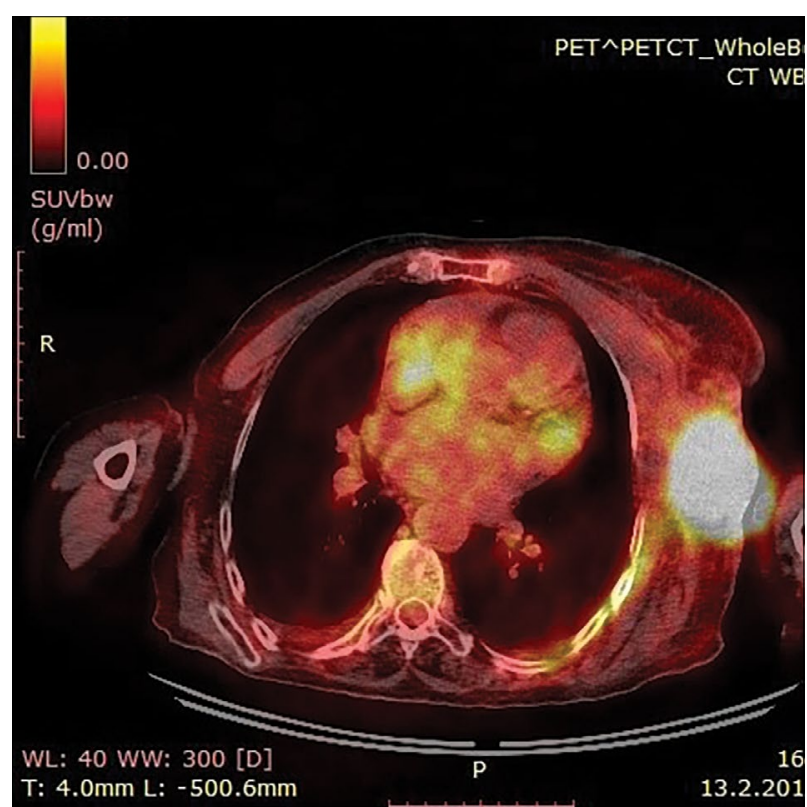

Fig. 3. PET image shows high uptake in the left axilla $\left(\mathrm{SUV}_{\max }: 16.69\right)$.

cytokeratin (CK)-7 and negativity for CK-20. These features resembled the original breast carcinoma. Thus, the tumor was not consistent with metastasis of the adenocarcinoma of the rectum. 


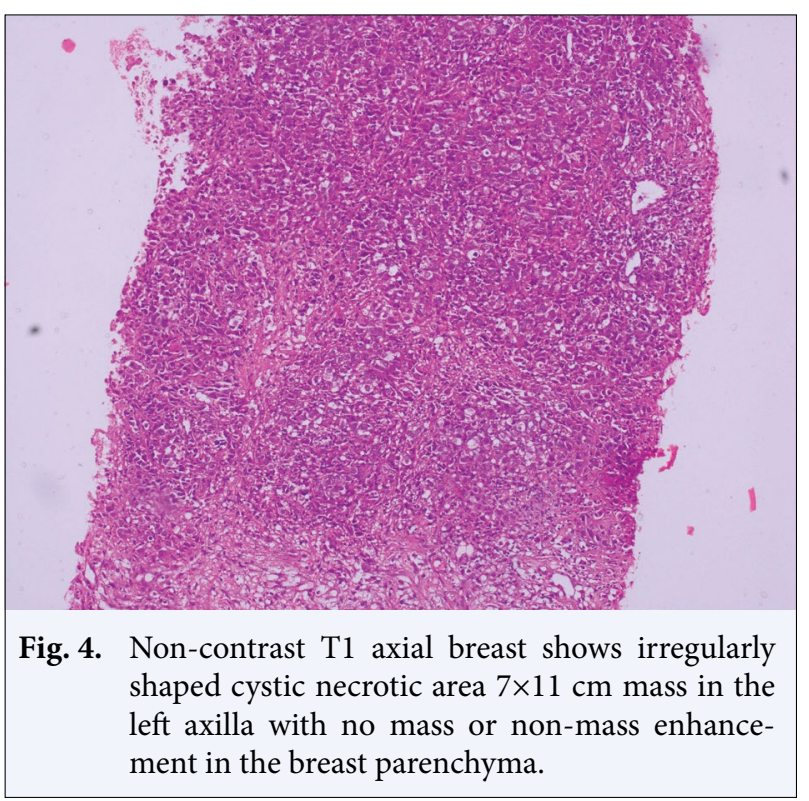

In March 2017, colonoscopy was performed, and an ulcerative, vegetative, $3-4 \mathrm{~cm}$ in size lesion was seen in the distal part of the rectum. Biopsy was obtained, and result was reported as adenocarcinoma of the rectum. Based on all the above findings, the outcomes of the patient were discussed in our hospital's oncology board, and the diagnosis was made as $\mathrm{OBC}$ with axillary metastatic carcinoma and synchronous rectum cancer. Radiotherapy (RT) was recommended because she was considered inoperable due to cardiovascular problems. Conformal RT was performed to the left breast, supraclavicular fossa, and axillary level I, II, and III regions with a total dose of $50 \mathrm{~Gy}$ with an additional total of 16 Gy boost to mass. During breast RT, the patient complained about rectal bleeding. Palliative RT was planned to the rectum to stop bleeding and applied with a daily fraction dose of $300 \mathrm{cGy}$ to a total dose of $30 \mathrm{~Gy}$. In the follow-up period, axillary mass nearly disappeared in 2 months, and she also had no complaints about rectal bleeding. After 3 months, the patient reported shortness of breath. Clinical examination and thorax CT showed that pulmonary edema occurred due to congestive heart failure, which was the reason of the patient's death.

\section{Discussion}

In general, palpable axillary nodes are more often related to benign rather than to malignant disorders. [6] Postmortem examinations showed that when cancer is identified, the origin of axillary lymphadenopathy in $55 \%-60 \%$ of the population with axillary mass is the breast, followed by the lymphomas, lungs, melanomas, thyroid cancers, and urogenital tract.[10] Performing comprehensive and systematic physical and imaging examinations is necessary to identify the primary origin. Studies of angiogenesis in the growth process and metastasis of OBC may show the primary carcinoma metastasis occurring at subclinical level.[11]

The National Comprehensive Cancer Network guidelines recommend MRI to identify occult breast lesions not identified by clinical examination or mammography. MRI showed high sensitivity (ranging from 94\% to $100 \%$ ) for the detection of OBC.[12] However, specificity has generally been lower and more variable and ranges from $37 \%$ to $97 \%$.[13]

Olson et al. compared the ability of MRI with traditional imaging methods for the diagnosis of OBC in 40 patients and found that MRI accurately locates the primary foci in resected specimens after breast surgery in 21 (95\%) out of 22 suspected cases. Of the 12 cases with negative MRI results, five underwent breast surgery, but no primary lesion was detected in four $(80 \%)$ cases. [14]

Owing to unknown clinical symptoms, $\mathrm{OBC}$ is usually detected later than other breast cancers. The diagnosis is usually made in later stages, and the tumor size is generally greater.[15] The assessment of histological examination is useful for the differential diagnosis of breast cancer. Morphological findings are the important diagnostic tool in daily clinical applications in the differential diagnosis of tumors in tissues; however, these may not be sufficient. The pathological examination should include IHC staining for ER, PR, HER2, CK-7, CK-20, alpha-lactalbumin, and GCDFP-15. For the following markers, GCDFP-15 is positive in $21 \%-55 \%$ of breast carcinomas and $56 \%-75 \%$ of breast carcinoma of apocrine histology.[16] Positive staining for CK-7 and negative staining for CK-20 support a possible breast carcinoma.[17] In the present case, IHC showed that the presence of GCDFP-15, CK-7 positivity, and CK-20 negativity provided us with the most important information to confirm the diagnosis with $\mathrm{OBC}$ by ruling out axillary metastasis from rectum cancer.

Axillary lymph node dissection (ALND) or RT is the treatment of choice in $\mathrm{OBC}$ with axillary metastasis; comparison of both approaches pointed to prognosis after ALND is better than RT.[18] All parents with isolated axillary metastasis should select ALND. However, in the present case, ALND could not be applied to the patient because of heart failure. RT was administered to the axillary region.

The optimal treatment of the ipsilateral breast is controversial. Options include mastectomy, breastconserving treatment using whole breast radiotherapy, 
and observation alone.[19,20] Breast without definitive local therapy is generally not recommended. Ipsilateral breast should be included in the RT treatment area due to increased cancer development risk.[21] In the present case, RT was performed in the ipsilateral breast in addition to the axilla.

The survival rates of patients with OBC compared with patient's with detectable tumors have been investigated in many studies. Rosen et al. declared that a group of patients with $\mathrm{OBC}$ has better outcomes than anatomic stage II-III with clinically apparent breast cancer although not statistically significant.[22,23]

Merson et al. reported that the 5- and 10-year survival rates among patients with $\mathrm{OBC}$ are $76.6 \%$ and $58.3 \%$, respectively. Subgroup analysis showed the number of involved lymph nodes associated with the prognosis and no difference in survival rates of women who had mastectomy and who underwent RT.[23]

In 1973, Browson et al. referred to tumors diagnosed at the same time or within 6 months as synchronous primary tumors and metachronous tumors diagnosed after 6 months depending on appearance time.

The incidence of MPTs is being frequently reported owing to new diagnostic techniques and longer survival rates of patients with cancer. The incidence of MPTs varies between $2.4 \%$ and $8 \%$ up to $17 \%$ in a cancer population within 20 years of follow-up.[24] MPTs have worse prognosis than other single primary tumors.[25]

In fact, there are responsible mechanisms, but the exact cause of MPTs has not been fully explained. Genetic susceptibility, the immune system of patients, and chemotherapy and RT used in the treatment of tumors are among the most common factors of MPTs.[26,27]

\section{Conclusion}

In conclusion, a review of the literature reveals that synchronous OBC and rectum cancer are extremely rare neoplasms. IHC should be analyzed in detail to distinguish between primary tumors and metastatic tumors. On the other hand, the present study demonstrated the importance of diagnostic and therapeutic strategies due to the worse prognosis of MPTs.

Informed consent: Written informed consent was obtained from the patient for the publication of the case report and the accompanying images.

Peer-review: Externally peer-reviewed.

Conflict of Interest: The authors declare that they have no conflict of interest.

Authorship contributions: Concept - Ö.A., L.S.; Design Ö.A., L.S.; Supervision - Ö.A., L.S., A.Y.Z.; Materials - Ö.A., B.K.O.; Data collection \&/or processing - Ö.A.B.K.O.; Anal- ysis and/or interpretation - Ö.A., A.Y.Z.; Literature search - Ö.A., L.S., B.K.O.; Writing - Ö.A.; Critical review - Ö.A., L.S., A.Y.Z.

\section{References}

1. Kebudi A. Occult Breast Cancer. The Journal of Breast Health 2013;9(3):118-24.

2. Amin MB, Edge SB, Greene FL, Schilsky RL, Byrd DR, Gaspar LE, et al., editors. AJCC cancer staging manual. 8th ed. Chicago; IL: Springer International publishing. 2017.

3. Takabatake D, Taira N, Aogi K, Ohsumi S, Takashima $\mathrm{S}$, Inoue $\mathrm{T}$, et al. Two cases of occult breast cancer in which PET-CT was helpful in identifying primary tumors. Breast Cancer 2008;15(2):181-4.

4. Baron PL, Moore MP, Kinne DW, Candela FC, Osborne MP, Petrek JA. Occult breast cancer presenting with axillary metastases. Updated management. Arch Surg 1990;125(2):210-4.

5. Xu LL, Gu KS. Clinical retrospective analysis of cases with multiple primary malignant neoplasms.Genet Mol Res 2014;13(4):9271-84.

6. Walsh R, Kornguth PJ, Soo MS, Bentley R, DeLong DM. Axillary lymph nodes: mammographic, pathologic, and clinical correlation. AJR Am J Roentgenol 1997;168(1):33-8.

7. Noh SK, Yoon JY, Ryoo UN, Choi CH, Sung CO, Kim TJ, et al. A case report of quadruple cancer in a single patient including the breast, rectum, ovary, and endometrium. J Gynecol Oncol 2008;19(4):265-9.

8. Lee JS, Moon W, Park SJ, Park MI, Kim KJ, Jang LL, et al. Triple synchronous primary cancers of rectum, thyroid, and uterine cervix detected during the workup for hematochezia. Intern Med 2010;49(16):1745-7.

9. Etiz D, Metcalfe E, Akcay M. Multiple primary malignant neoplasms: A 10-year experience at a single institution from Turkey. J Cancer Res Ther 2017;13(1):16-20.

10. Lloyd MS, Nash AG. 'Occult' breast cancer. Ann R Coll Surg Engl 2001;83(6):420-4.

11. Locopo N, Fanelli M, Gasparini G. Clinical significance of angiogenic factors in breast cancer. Breast Cancer Res Treat 1998;52(1-3):159-73.

12. Orel SG, Schnall MD, LiVolsi VA, Troupin RH. Suspicious breast lesions: MR imaging with radiologicpathologic correlation. Radiology 1994;190(2):485-93.

13. Orel SG. MR imaging of the breast. Radiol Clin North Am 2000;38(4):899-913.

14. Olson JA Jr, Morris EA, Van Zee KJ, Linehan DC, Borgen PI. Magnetic resonance imaging facilitates breast conservation for occult breast cancer. Ann Surg Oncol 2000;7(6):411-5.

15. Oger AS, Boukerrou M, Cutuli B, Campion L, Rousseau E, Bussières E, et al. Male breast cancer: prognostic factors, diagnosis and treatment: a multiinstitutionalsurvey of 95 cases. Gynecol Obstet Fertil 2015;43(4):290-6. 
16. Rosen PP. Rosen's Breast Pathology. 2nd edition. Philadelphia: Lippincott Williams \& Wilkins; 2001. p. 493.

17. Ünlü M, Canda T, Sevinç A, Harmancıoğlu Ö, Saydam S, Koçdor MA, et al. Primer ve metastatik meme kanserlerinde GCDFP-15, ALA, SK7 ve SK20 boyanma özellikleri. Türk Patoloji Dergisi 2009;25(3):78-84.

18. Rosen PP, Kimmel M. Occult breast carcinoma presenting with axillary lymph node metastases: a follow-up study of 48 patients. Hum Pathol 1990;21(5):518-23.

19. Feigenberg Z, Zer M, Dintsman M. Axillary metastases from an unknown primary source. Isr J Med Sci 1976;12(10):1153-8.

20. Vilcoq JR, Calle R, Ferme F, Veith F. Conservative treatment of axillary adenopathy due to probable subclinical breast cancer. Arch Surg 1982;117(9):1136-8.

21. Ellerbroek N, Holmes F, Singletary E, Evans H, Oswald M, McNeese M. Treatment of patients with isolated axillary nodal metastases from an occult primary carcinoma consistent with breast origin. Cancer 1990;66(7):1461-7.
22. Kemeny MM, Rivera DE, Terz JJ, Benfield JR. Occult primary adenocarcinoma with axillary metastases. Am J Surg 1986;152(1):43-7.

23. Merson M, Andreola S, Galimberti V, Bufalino R, Marchini S, Veronesi U. Breast carcinoma presenting as axillary metastases without evidence of a primary tumor. Cancer 1992;70(2):504-8.

24. Vogt A, Schmid S, Heinimann K, Frick H, Herrmann C, Cerny $\mathrm{T}$, et al. Multiple primary tumours: challenges and approaches, a review. ESMO Open 2017;2(2):e000172.

25. Yılmazer G, Doğan E, Nart M. Multiple Primer Tümör: Olgu Sunumu. Van Tip Dergisi 2015;22(2):118-21.

26. Ferlay J, Steliarova-Foucher E, Lortet-Tieulent J, Rosso S, Coebergh JW, Comber H, et al. Cancer incidence and mortality patterns in Europe: estimates for 40 countries in 2012. Eur J Cancer 2013;49(6):1374403.

27. Kim JY, Song HS. Metachronous double primary cancer after treatment of breast cancer. Cancer Res Treat 2015 Jan;47(1):64-71. 